\title{
Low-mass dielectron measurements with ALICE at the LHC
}

\author{
Raphaelle Bailhache ${ }^{1, *}$ for the ALICE Collaboration \\ ${ }^{1}$ Goethe Universitaet Frankfurt-am-Main, Germany
}

\begin{abstract}
Recent measurements of $\mathrm{e}^{+} \mathrm{e}^{-}$pair production in $\mathrm{pp}$ and $\mathrm{p}-\mathrm{Pb}$ collisions at the center-of-mass energy $\sqrt{s}_{\mathrm{NN}}=5.02 \mathrm{TeV}$ are reported. Cold nuclear matter effects such as shadowing, as well as the possible presence of thermal radiation, are investigated in $\mathrm{p}-\mathrm{Pb}$ collisions with the dielectron nuclear modification factor $R_{\mathrm{pPb}}$. Furthermore, results on dielectrons at low $p_{\mathrm{T}, \mathrm{ee}}$ in peripheral $\mathrm{Pb}-\mathrm{Pb}$ collisions at $\sqrt{s}_{\mathrm{NN}}=5.02 \mathrm{TeV}$ and in pp collisions at $\sqrt{s}=13 \mathrm{TeV}$ are presented and compared to calculations.
\end{abstract}

\section{Introduction}

Dileptons and photons are unique tools to study the space-time evolution of the hot and dense matter created in ultra-relativistic heavy-ion collisions. They are produced during the entire history of the collision with negligible final state interaction. Therefore they carry undistorted information since the early stages of the collision. On top of the hadronic interactions, dielectrons can be produced in initial photon annihilation processes, triggered by the coherent electromagnetic (EM) fields of the incoming nuclei. The latter can be used to map the very strong, varying EM fields produced by the passing nuclei.

The data shown in this contribution were recorded with the ALICE experiment at the LHC during proton-proton $(\mathrm{pp})$, proton-lead $(\mathrm{p}-\mathrm{Pb})$ and lead-lead $(\mathrm{Pb}-\mathrm{Pb})$ runs at a nucleonnucleon center-of-mass energy $\sqrt{s}_{\mathrm{NN}}=5.02 \mathrm{TeV}$ and during a campaign with a reduced magnetic field of the ALICE solenoid in pp collisions at $\sqrt{s}=13 \mathrm{TeV}$. Electrons are tracked and identified at mid-rapidity $\left(\left|\eta_{\mathrm{e}}\right|<0.8\right)$ in the central barrel with the Inner-Tracking-System (ITS), the Time Projection Chamber (TPC) and the Time-Of-Flight detector.

\section{Dielectrons in pp and $\mathrm{p}-\mathrm{Pb}$ collisions at $\sqrt{s}_{\mathrm{NN}}=5.02 \mathrm{TeV}$}

First, the measurement of the dielectron production in pp collisions at $\sqrt{s}=5.02 \mathrm{TeV}$ is shown on the left panel of Fig. 1 and constitutes an important baseline for studies in $\mathrm{p}-\mathrm{Pb}$ and $\mathrm{Pb}-\mathrm{Pb}$ collisions at the same $\sqrt{s}_{\mathrm{NN}}[1]$. The results, obtained for a transverse momentum of the electrons $p_{\mathrm{T}, \mathrm{e}}>0.2 \mathrm{GeV} / c$, are fully described by the expectations from known hadronic sources, i.e. the hadronic cocktail. Independent measurements of mesons are taken as input to compute the dielectron cross sections from light-flavour hadron and $J / \psi$ decays, whereas the contributions from $\mathrm{c} \overline{\mathrm{c}} \rightarrow \mathrm{e}^{+} \mathrm{e}^{-}$and $\mathrm{b} \overline{\mathrm{b}} \rightarrow \mathrm{e}^{+} \mathrm{e}^{-}$are calculated with the POWHEG event generator and fitted to data in the dielectron invariant mass $m_{\mathrm{ee}}$ region $1.1<m_{\mathrm{ee}}<2.7 \mathrm{GeV} / c^{2}$. The dielectron cross-section in $\mathrm{p}-\mathrm{Pb}$ collisions is directly compared to the measurement in

\footnotetext{
*e-mail: raphaelle.bailhache@cern.ch
} 

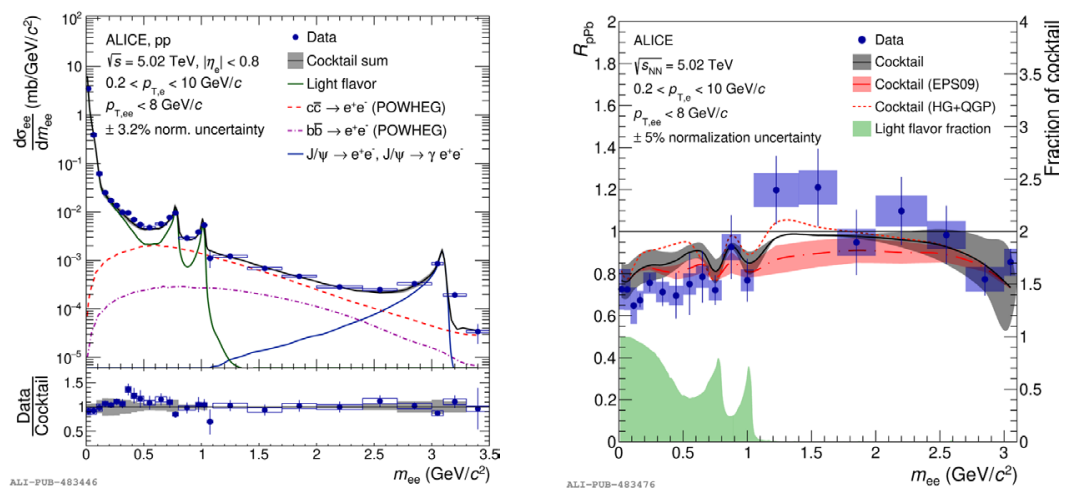

Figure 1. Left: dielectron production in pp collisions at $\sqrt{s}=5.02 \mathrm{TeV}$ compared to the expectations from known hadronic decays [1]. Right: dielectron nuclear modification factor in $\mathrm{p}-\mathrm{Pb}$ collisions at $\sqrt{s}_{\mathrm{NN}}=5.02 \mathrm{TeV}$ compared to calculations [1].

pp collisions at the same $\sqrt{s}_{\mathrm{NN}}(=5.02 \mathrm{TeV})$ via the dielectron nuclear modification factor $R_{\mathrm{pPb}}$ on the right panel of Fig.1 [1]. The $m_{\mathrm{ee}}$ dependence of $R_{\mathrm{pPb}}$ reflects the different scaling behaviours of the light-flavour $\left(m_{\mathrm{ee}}<1 \mathrm{GeV} / c^{2}\right)$ and heavy-flavour $\left(m_{\mathrm{ee}}>1 \mathrm{GeV} / c^{2}\right)$ productions. The results are compared to $c \bar{c}$ calculations including nuclear shadowing with the EPS09 nPDF and predictions including thermal radiation from the medium [1]. Both are disfavoured by the data in different $m_{\mathrm{ee}}$ ranges, i.e. $1.1<m_{\mathrm{ee}}<2.7 \mathrm{GeV} / c^{2}$ and $m_{\mathrm{ee}}<0.5 \mathrm{GeV} / c^{2}$, respectively. Future analyses as a function of the distance-of-closest approach (DCA) to the primary vertex of the $\mathrm{e}^{+} \mathrm{e}^{-}$pairs, as well as the charged-particle multiplicity in the event, would give the possibility to study possible prompt thermal contributions, separated from the heavy-flavour contribution.

\section{Dielectrons in peripheral $\mathrm{Pb}-\mathrm{Pb}$ collisions at $\sqrt{s}_{\mathrm{NN}}=5.02 \mathrm{TeV}$}

The preliminary results on the dielectron yield, integrated over $p_{\mathrm{T}, \mathrm{e}}$, in semi-peripheral (50$70 \%$ ) and peripheral (70-90\%) central $\mathrm{Pb}-\mathrm{Pb}$ collisions at $\sqrt{s}_{\mathrm{NN}}=5.02 \mathrm{TeV}$ are compatible with cocktails of known hadronic sources, as can be seen on the left panel of Fig.2. The data are nevertheless also in agreement with calculations including thermal radiations from the medium [2]. For $p_{\mathrm{T}, \mathrm{ee}}<0.1 \mathrm{GeV} / c$, the vacuum hadronic cocktails clearly underestimate the measured $\mathrm{e}^{+} \mathrm{e}^{-}$yields shown on the right panel of Fig.2. The overshoot is more significant in more peripheral $\mathrm{Pb}-\mathrm{Pb}$ collisions. The measured $p_{\mathrm{T}, \text { ee }}$ excess spectrum in $0.4<m_{\mathrm{ee}}$ $<0.7 \mathrm{GeV} / c^{2}$ for peripheral $\mathrm{Pb}-\mathrm{Pb}$ collisions, in the left panel of Fig.3, is fairly well reproduced by predictions for the coherent photoproduction of dielectrons $\left(\gamma \gamma \rightarrow \mathrm{e}^{+} \mathrm{e}^{-}\right)$using the Wigner distribution formalism to take into account the impact parameter dependence of the electromagnetic fields [3]. This process leads to a $m_{\mathrm{ee}}$ continuum of very soft $\mathrm{e}^{+} \mathrm{e}^{-}$pairs in $p_{\mathrm{T} \text {,ee }}$, whose centrality dependence is much milder than that of the known hadron decays or thermal radiations in hadronic collisions. Therefore its relative contribution increases in peripheral collisions.

\section{Soft dielectron production in pp collisions at $\sqrt{s}=13 \mathrm{TeV}$}

In the past decades, several experiments reported an anomalous soft-photon and dilepton excess in hadron-hadron collisions [4]. In pp collisions at $\sqrt{s}=13 \mathrm{TeV}$, a dedicated data- 

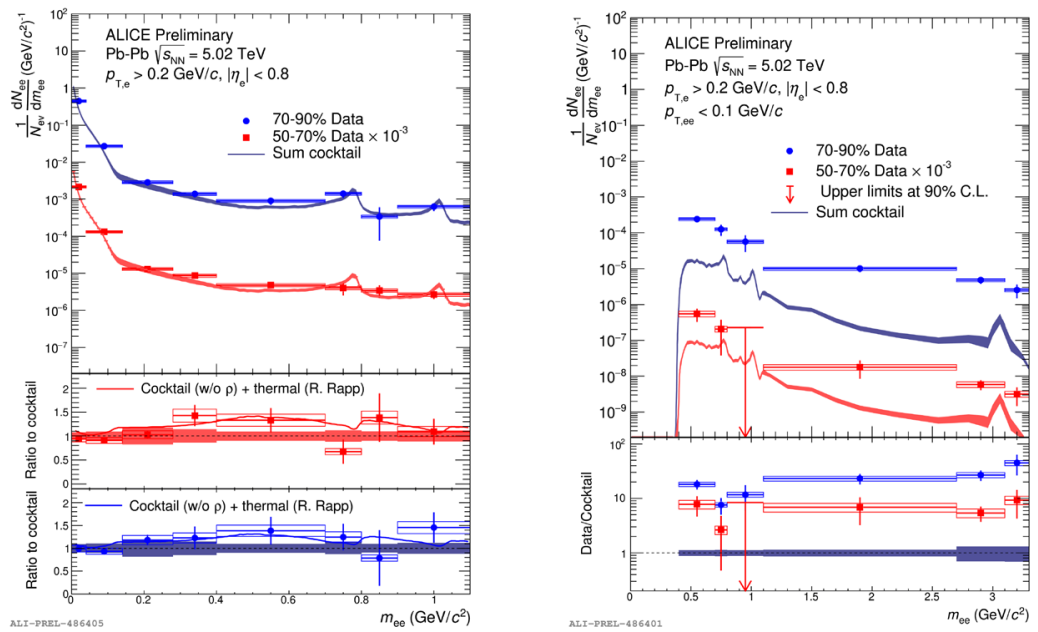

Figure 2. Dielectron yields in semi-peripheral (50-70\%) and peripheral (70-90\%) $\mathrm{Pb}-\mathrm{Pb}$ collisions integrated over $p_{\mathrm{T}, \mathrm{ee}}$ (left panel) and for $p_{\mathrm{T}, \mathrm{ee}}<0.1 \mathrm{GeV} / c$ (right panel) compared to the hadronic cocktail including or not thermal radiations.
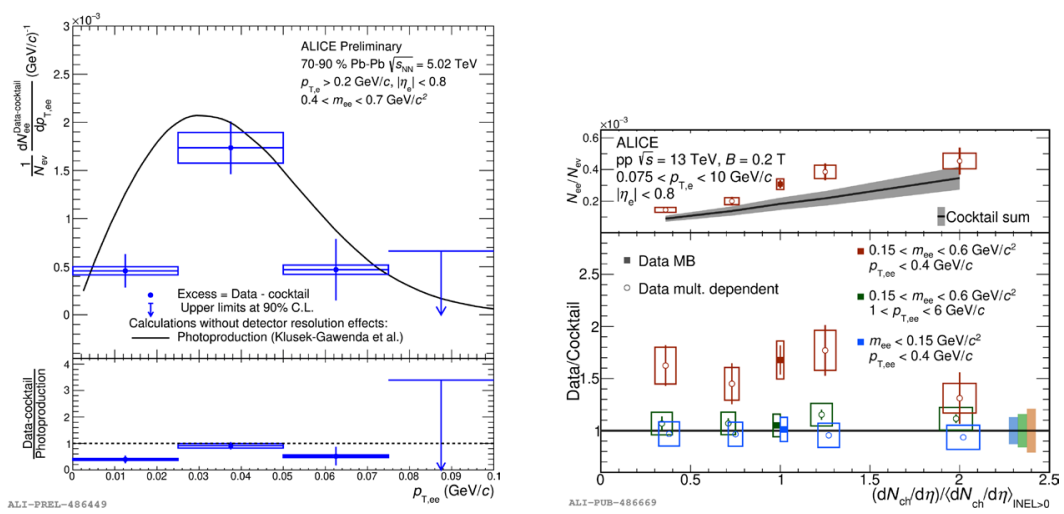

Figure 3. Left: excess dielectron yield as a function of $p_{\mathrm{T} \text {,ee }}$ in $0.4<m_{\mathrm{ee}}<0.7 \mathrm{GeV} / c^{2}$ for peripheral $\mathrm{Pb}-\mathrm{Pb}$ collisions compared to calculations for $\gamma \gamma \rightarrow \mathrm{e}^{+} \mathrm{e}^{-}$[3]. Right upper panel: $\mathrm{e}^{+} \mathrm{e}^{-}$yield in $\mathrm{pp}$ collisions at $\sqrt{s}=13 \mathrm{TeV}$ in the excess region as a function of the event multiplicity compared with the hadronic cocktail. Right lower panel: enhancement factor data/cocktail in pp collisions at $\sqrt{s}=13$ $\mathrm{TeV}$ for three different kinematic regions [4].

taking campaign with a reduced magnetic field of the ALICE solenoid $(0.2 \mathrm{~T})$ allows the study of soft $\mathrm{e}^{+} \mathrm{e}^{-}$production $\left(p_{\mathrm{T}, \mathrm{e}}>75 \mathrm{MeV} / c\right)$, previously inaccessible at the LHC. The measured dielectron cross section is above the expectations from the known hadronic decays by a factor $1.61 \pm 0.13$ (stat.) \pm 0.17 (syst.) \pm 0.34 (cocktail) in $0.14<m_{\mathrm{ee}}<0.6 \mathrm{GeV} / c^{2}$ for $p_{\mathrm{T}, \mathrm{ee}}<0.4 \mathrm{GeV} / c$ [4]. In the right upper panel of Fig.3, the multiplicity dependence of the dielectron yield is shown in orange and compared to the one of the hadronic cocktail. No clear multiplicity dependence of the data-to-cocktail ratio is observed within the uncertainties. For comparison, this ratio is also plotted for lower $m_{\mathrm{ee}}$ (blue) or higher (green) $p_{\mathrm{T}, \mathrm{ee}}$, where no excess is found. The excess of the acceptance corrected yield can be reproduced neither by 


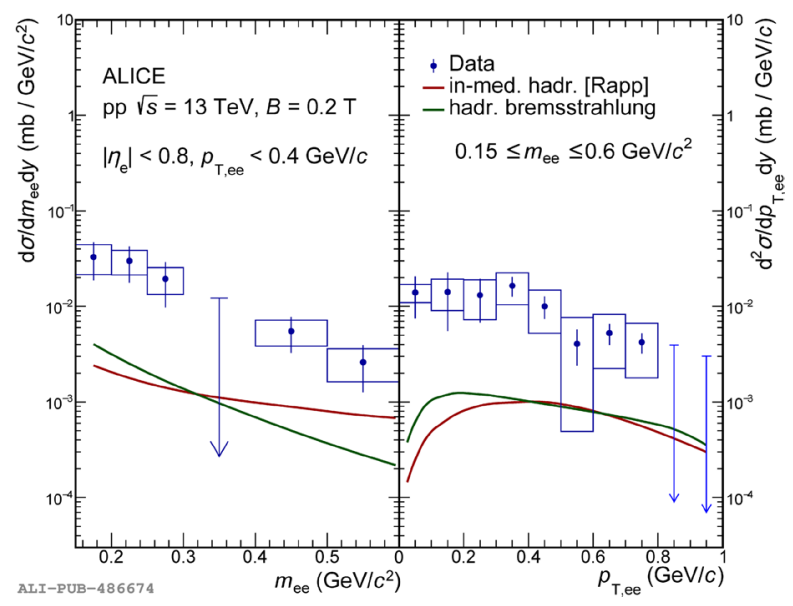

Figure 4. Dielectron excess spectra as a function of $m_{\mathrm{ee}}$ (left) and $p_{\mathrm{T}, \mathrm{ee}}$ (right) compared to calculations of bremsstrahlung from initial- and final-state hadrons, and thermal dielectron production in $\mathrm{pp}$ collisions at $\sqrt{s}=13 \mathrm{TeV}[4]$.

calculations for bremsstrahlung from initial- and final-state hadrons, nor by prediction for thermal dielectron production [4], as can be seen in Fig.4. A possible mechanism recently proposed to explain the data is a back-reaction of the QCD vacuum to non-perturbative $q \bar{q}$ pairs [5], only visible with real or virtual photons.

\section{Summary and outlook}

The measured dielectron cross section in pp collisions at $\sqrt{s}=5.02 \mathrm{TeV}$ for $p_{\mathrm{T}, \mathrm{e}}>0.2 \mathrm{GeV} / c$ provides a well-understood baseline for $\mathrm{p}-\mathrm{Pb}$ and $\mathrm{Pb}-\mathrm{Pb}$ studies at the same center-of-mass energy. In $\mathrm{p}-\mathrm{Pb}$ collisions at $\sqrt{s_{\mathrm{NN}}}=5.02 \mathrm{TeV}$, no significant modification of the heavyflavour production is observed within the experimental uncertainties. Analyses as a function of the pair DCA will help to disentangle the heavy-flavour contribution from possible thermal radiations. In peripheral $\mathrm{Pb}-\mathrm{Pb}$ collisions at $\sqrt{s_{\mathrm{NN}}}=5.02 \mathrm{TeV}$, the measured dielectron yields clearly overshoot the hadronic cocktail for $p_{\mathrm{T} \text {,ee }}<0.1 \mathrm{GeV} / c$. The excess is fairly well reproduced by calculations for coherent photoproduction of $\mathrm{e}^{+} \mathrm{e}^{-}$pairs. Analyses of highstatistics central $\mathrm{Pb}-\mathrm{Pb}$ collisions are still ongoing. In pp collisions at $\sqrt{s}=13 \mathrm{TeV}$, the soft dielectron production $\left(p_{\mathrm{T}, \mathrm{e}}>0.75 \mathrm{GeV} / c\right)$ is measured for the first time at LHC energies. An enhancement over the hadronic cocktail is found, whose multiplicity dependence is still inconclusive. Thanks to the ALICE ITS and TPC upgrades in Run 3 and 4, the DCA resolution and the readout rate will be significantly improved, leading, for example, to an increase of statistics by a factor 400 for soft-dielectron studies in pp collisions [6].

\section{References}

[1] ALICE Collaboration, Phys. Rev. C102, 055204 (2020)

[2] R. Rapp, Adv. High Energy Phys. 2013, 148253 (2013)

[3] M.Klusek-Gawenda et al., Phys. Lett. B814, 136114 (2021)

[4] ALICE Collaboration, Phys. Rev. Lett. 127, 042302 (2021)

[5] O. Garcia-Montero, arXiv:2104.07050

[6] ALICE Collaboration, CERN-LHCC-2020-018 